\title{
The structure design of electronic finder under the office
}

\author{
Hu Wenfei
}

\author{
Wenzhou Vocational \& Technical College, Wenzhou. 325035 \\ 61230934@qq.com
}

Keywords: Office; searching; study; product .

\begin{abstract}
There are a number of folders at today's workplace. Although some people will spend time to arrange them neatly, when the quantity is abundant, it is not easy to identify them from a lot of files. The workers are being affected by these complicated problems. Even small problems sometimes are annoying. To solve this problem and improve the work efficiency, we need to create a kind of future office supply. Relying on excellent technology and by means of document retrieval, this product is not only easy to operate but also can find the files effectively. So, this design is especially for the group of white-collar workers working at the office. They read all kinds of files every day and definitely will directly be affected by such problems. Undoubtedly, this new design can alleviate some of their work pressure.
\end{abstract}

\section{Introduction}

According to the principle of electromagnetic wave reflection (specifically see design patent of Qian weidong) used in public office, anti-theft products are made by magnetic stripe as a reference sample. Firstly, users should divide the items according to their important level; secondly, label them with different tags and input the data latch into the finder to register and summarize the information of items. If users do not remember the locations of items, it will be easy to find them in the short distance of the workplace. Such product is not only designed for lazy people, but also truly conforms to the principle of people-oriented. Its true purpose is to provide convenience for people. With it, people will greatly improve the work efficiency, and increase the fun of work. From now on, you never need to be panic for missing a file.

\section{Related influence factors of electronic anti-lost device (security products and security alarm equipments)}

Generally speaking nowadays, only four aspects are considered to evaluate an innovative enterprise in China: (1)the technology advancement, feasibility and continuity; (2)economic effect; (3) social effect; (4)the future availability. My graduation design also considers factors mentioned above to ensure this product with more feasibility and practical, at the same time, to increase the value of this study.

1) This electronic finder can be seen as a solution to a series problems daily office involved. Such as: the product durability, color, appearance, materials and even some characteristics of science and technology.

2) The application of some new structures and technologies. I considered the application of new materials, locking and security settings of this product, and also considered its environmental protection, recycling and disassemble possibility.

3) Studying the best ways of operation for the electric finder, positioning its function, solving the problems of technology and structure, realizing the functions finally. See picture1. I put forward several design schemes, and select the best one to satisfy the requirements of graduation design. 


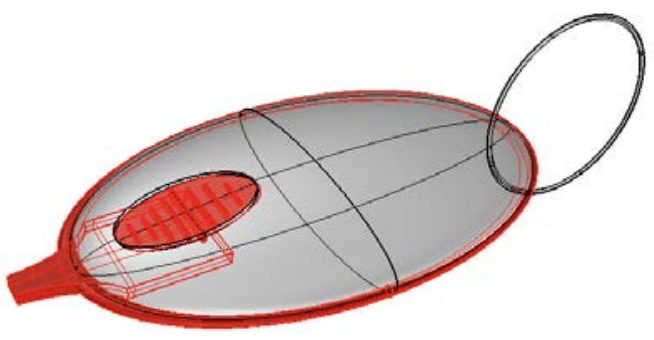

Picture1.The structure analysis

4) The goals should be achieve from the final scheme.

\section{Working mode analysis}

This electronic finder system adopts operating procedures by two steps. Firstly, marking the important documents as data clip in advance, and recording the data into the hard disk of the host, then putting the clamps on the folder. Secondly, when you want to search for a folder or folders, as long as turning on the host of the electronic finder, inputting key information into the color screen interface and sending out the searching signal, the general orientation of the files will be shown on the screen. At the same time, the clip as a receiver will send out the sound that is easily identified as a hint. Through this product, it is easy to achieve the goals of working conveniently and efficiently.

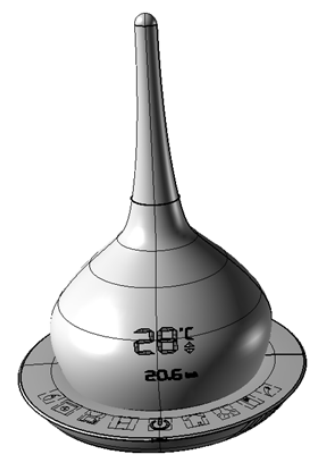

Picture2. The host structrue

The content mentioned above is the case in operation. When the machine is in a state without use, the host is placed on the outlet, at this moment, the device is shutdown into dormancy automatically and into energy saving mode, which is beneficial to save resources and avoid waste. Besides, the host has a fixed place to stay not only prevent from missing but also play the role of protecting the device against breaking.

Generally speaking, the electronic finder is a kind of device with high technology, continuity and with strong purposeful work mode. The users do not need too much of professional training. All operating prompt will be shown on the screen panel, so it can help users finish their work easily and conveniently.

\section{Material analysis}

The shell material is polyformaldehyde. Polyformaldehyde is a kind of polymer, referring to the macromolecular chain containing methylene oxide repeating structural unit, known as poly methylene oxide. Its English name is Polyacetal or Polyoxymethylene, hereinafter short for as POM. POM is the third general engineering materials used mostly. According to different structures, POM can be divided into two kinds of homopolymerization POM and copolymerization POM POM. Homopolymerization POM is synthesized by formaldehyde or melamine formaldehyde as the raw material. Repeating structural unit of Macromolecular chain is methylene oxide. In order to improve its heat resistance, $\mathrm{i}$ uses ester base or ether base to seal sides. 
The advantages of POM: Its mechanical properties and good rigidity are close to the metal material. It is an ideal substitute material for copper, zinc, steel, aluminum and other metal materials. Its fatigue resistance and creep resistance are excellent. And its resistance to wear, the friction are good too. POM as well as UHMWPE, PA, F4 is called four wear-resistant materials. It is also with high thermal stability, chemical stability and good electrical insulation.

The disadvantages of POM are: big density, poor acid resistance and heat resistance, later contracting is large and unstable, poor dimension stability.

POM is widely used in electronics/electrical appliances, machineries, automobiles, instruments/meters, buildings and daily necessities, etc. Different areas with different emphasis, such as Japan $40 \%$ used for electrical/electronics, $27 \%$ used for automobiles, $14 \%$ used for machineries. In the United States $45 \%$ use for electronics/electrical equipment, $17.5 \%$ used for automobiles. In Western Europe39\% used for cars, 16\% used for electronics/electrical appliances.

Other parts of material, LCD external display screen transparent lens: PPMA

LCD internal display screen transparent lens: PPMA

Charging holder and side key: ABS

\section{Color analysis}

\subsection{The analysis of white}

White is the main tonal for this design in order to match the environment in the office of elegant, quiet, scientific and technologic. White color sends out the experience of pure, fresh, elegant and quiet, giving the white-collar workers a comfortable working environment. In commercial design, white means advanced and scientific, it is usually mixed with other colors. White is soft, elegant, and it belongs to middle gender, acceptable to both male and female. As we all know, white is never out of date, and is one of the eternal popular colors. People use white to convey the image of science and technology. The color white is mostly combined with a small amount of other colors to avoid the feeling of too dull, plain or stiff. In general, pure white feels cold, serious. When using white, it should add some other colors, such as ivory white, rice white, milky white, white apple and so on.

\subsection{The analysis of black}

In the commercial design, black means noble, steady, scientific and technologic. Many technology products mostly use black as staple colors, such as TV, sports car, camera, audio, the instrument. Among other things, black represents the image of solemn. The black is also commonly used in the space design of some special occasions. For example, the articles for daily use and dress design mostly use black to shape the image of the noble. Black is also a kind of eternal popular color, suitable for mixing with many colors.

\subsection{The analysis of orange}

Orange is with high visibility. Because of this characteristic, it is widely used as industrial safety color such as locomotive, mountaineering clothing, bags, jackets etc. Due to orange is a very bright and dazzling, it gives people a negative vulgar image sometimes, especially on the use of the dress. So, the use of orange should be more thoughtful. In order to perform properly its bright and lively flavors, it is necessary to pay attention to its collocation of colors and forms.

\subsection{Conclusion of collocation}

The collocation of black and white is the mainstream colors of electronic products nowadays, especially for high-end electronic products. The body of the white meets the development trend for e-commerce products. The use of black not only can have the effect of the segmentation module, but also can reach certain modification function. Collocation of black and white gives people a feeling of noble. With a small amount of bright color such as orange, it can make the design outstanding. The interface of the screen is adopted bright orange as the main color tonal in order to express the vigor of the whole design. See picture 3. 


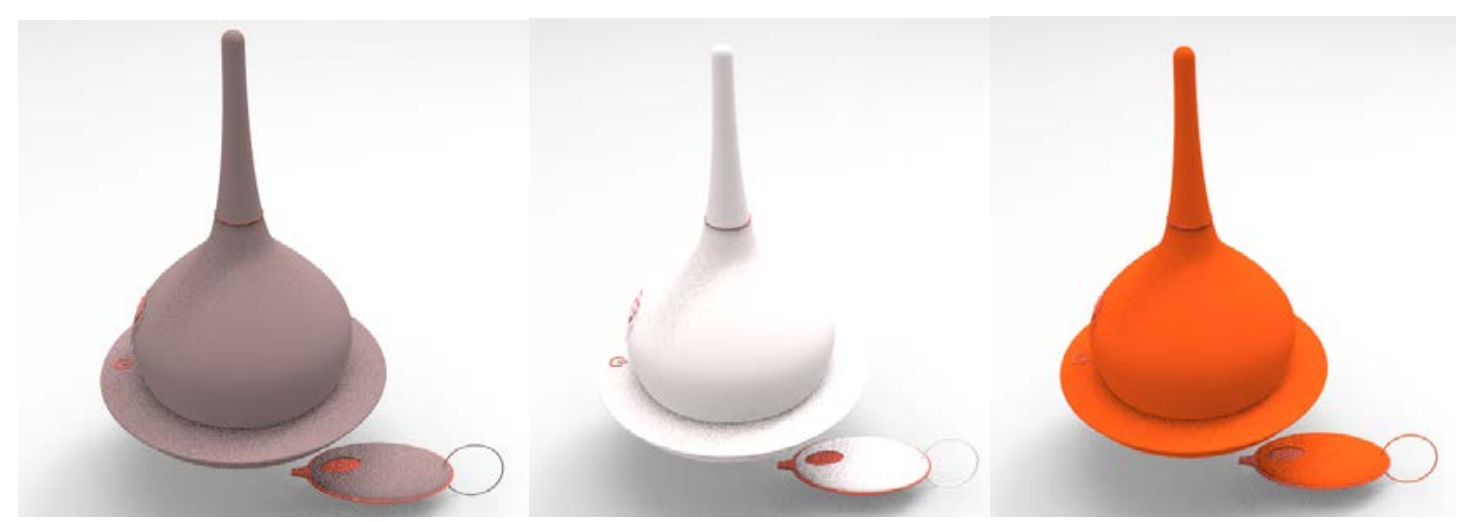

Picture3. The colors comparison

\section{Ergonomics analysis}

The concept of the people-oriented has influenced the modern fast development society in a variety ways, involving science and technology, the status of the family and all walks of life. Ergonomic is a design method which fully considers the human capabilities and limitations into a design, as shown in picture 4 . This helps to ensure the product to meet the needs of target consumers.
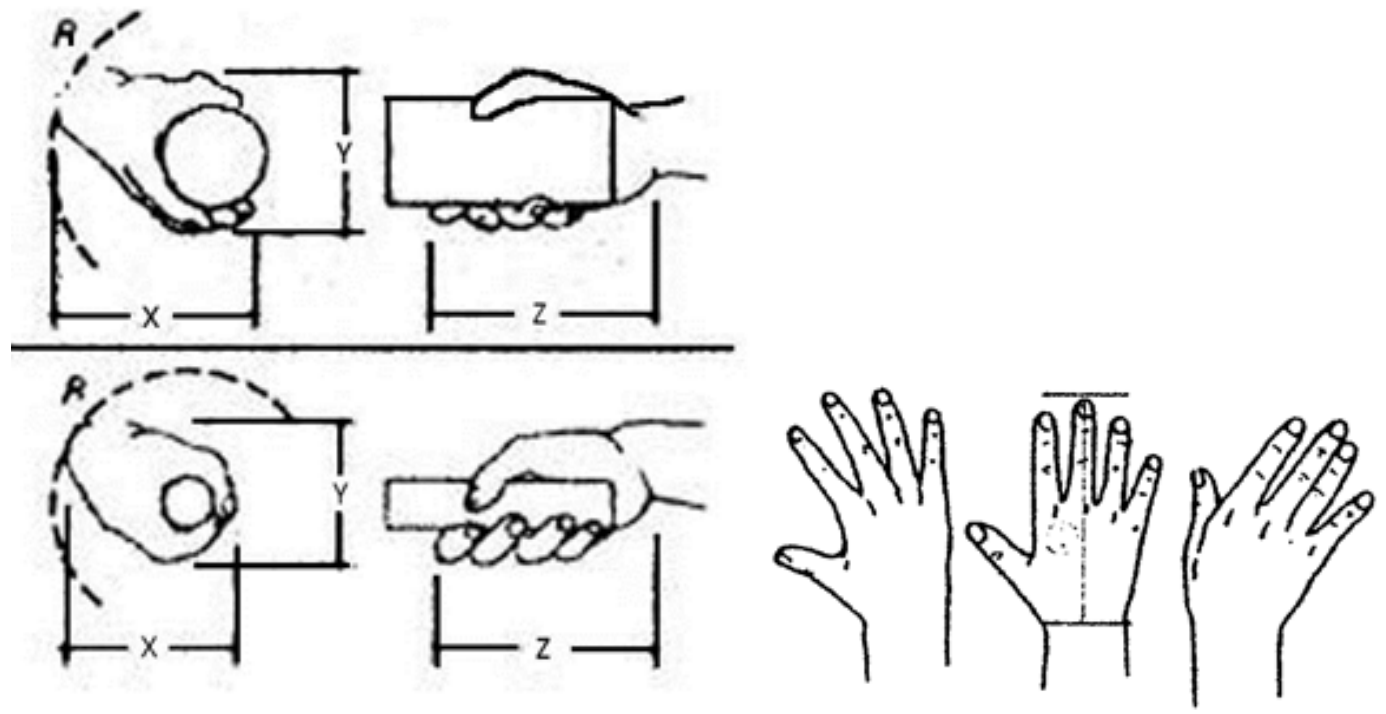

Picture4. Ergonomics analysis of hands

Scheme design should consider the relationship between products and surrounding environment, avoiding conflict and keeping products harmonious with the surrounding environment. The design of the product should be integrated into the work environment. It needs to consider the collocation of colors, office environment and the durability of materials. Besides, during the modeling design process, I also take into account that how to help users use the device more conveniently. Not only should it be comfortable, reducing hand fatigue from the long time use, but also easier to use with touching screen operation interface. In the interface design, the size and shape of the button settings are not only easy to recognize, preventing the wrong operation and looks more beautiful, but also satisfy users' the requirements of physiological and psychological.

Ergonomics analysis plays the most important role in this design. Careless consideration of ergonomics analysis not only affects users' operation, but also damages the whole work environment, sometime even delays the progress of future development. So, the correct man-machine analysis is a non-ignorable part for the whole design process.

\section{Summary}

With the improvement of material life, people's demands for higher quality of life are increasing at the same time. In order to purse more comfortable life, they do not mind spending a large number of 
money for it. As reported, the investment ratio for the quality of life has increased extremely. The functions of the product is not the only way to satisfy people's requirements at present, more and more people start to pay more attention on the visual aesthetic and mental pleasure from the products. So the purpose of the design should be people orientated and useful. My design is based on this purpose. On the basis of existing products and through design method, I studied the product innovation, form design, human-computer interaction, material selection, structure design, model making etc

\section{References}

[1] JIANG Nan, LI Wen-fei.The Humanity Design of a Foldable Walker[J].Journal of Engineering Graphics, 2010 (4) : 137-141.

[2] WILLIAM L.Universal Principles of Design[M].LI Chan, Translate.Shenyang : Liaoning Science and Technology Press, 2010.

[3] QIU Zi-hua, XI Chuan-jin.Unique Aesthetic Thinking System and Poetic Aesthetic Theory: On General Characters of the Oriental Aesthetics[J].Journal of Nanjing University, 2003 (1) : $106-116$.

[4] ZHANG Xin-qi.On Dynamic Design of Furniture: the Types of Folding Structure[J].Furniture \& Interior Design, 2007 (9) : 21-23.

[5] ZHANG Fu-chang, ZHANG Han-ning.Folding \& Folding Furniture[J].Furniture, 2002 (4) : 13-19. 